\title{
Phytochemical Nanomodification: Impending Perspectives
}

\section{Anjali Singh*}

Department of Pharmaceutical Medicine, Jamia Hamdard University, New Delhi, India

Constructive amalgamation of nature and science has enabled the humanoid specie to exploit the plant kingdom for its diverse purposes, major being nutritional and therapeutic needs. Advancements in science and technology have brought us to this era where complete automization of almost all the aspects of drug discovery to drug development and drug delivery can be achieved [1]. Recent studies on various indigenous and exotic plant species by different research groups around the world have revealed some major unidentified pharmacological actions that were not known before. Some of these chemical groups possessing significant pharmacological properties include polyphenols (Resveratrol), benzoquinones (Thymoquinone), flavonoids, steroids (Digoxin) and alkaloids (Vincristine), naphthaquinones (Lawsone) and anthraquinones (Sennosides), among others [2]. However, the unanimous snags of using phytochemical agents for therapeutic purposes are their poor aqueous solubility, photostability and subsequent absorption in the body, non-targeted drug distribution as well as low safety margin which collectively hampers the biopharmaceutical aspects of drug delivery viz pharmacokinetics and pharmacodynamics. A remarkably low drug distribution is observed while calculating the AUC (area under curve), a characteristic parameter in biopharmaceutics [3].

Nanostructures of different sizes, shapes and material properties have numerous applications in biomedical imaging, clinical diagnostics and therapeutics. Nanotechnological approaches can considerably control the drug release from the designed dosage form as well as the therapeutic index by enhancing localization in target tissues [4]. Nanomodification of phytotherapeutics (1-100 $\mathrm{nm}$ in at least one dimension) can significantly increase the dissolution of poorly soluble drugs, in addition to boosting their stability, bioavailability and decreasing their toxicity. Rationally designed nanocarriers with controlled key properties such as size, surface characteristics and the targeting moiety can revolutionize the use of phytochemicals for treatment of various ailments [5]. Reduction of particles to nano range alters the biodistribution characteristics of the formulation as well. Nanoparticles $(\sim 100-200 \mathrm{~nm})$ cross anatomical barriers by passing through small fenestrations present in the cell lining of each organ which is distinct in its own way [6]. For example, blood brain barrier is intact and has no fenestrations while rat liver has passages of upto $230 \mathrm{~nm}$ in their endothelial lining. Based on this principle, our group had constructed solid-lipid based nanoparticles in a matrix-type monolithic controlled delivery dosage form for delivering Thymoquinone to rat liver [7]. We observed significant improvements in drug release, pharmacokinetic and biodistribution profiles of the drug as compared to the conventional dosage form (suspension). A similar analysis was also conducted on nanomodified Resveratrol (study under review). Also, our group has worked on fabrication of colon-targeted microspheres of 5-FU using Assam bora rice starch as an important excipient and obtained significantly successful results [8].

Surface modification and/or functionalization of nanocarriers are a step forward which can be employed for effective targeting of the drug to the specific site of action. Thus, a crucial and rational control of nanoparticle size can enhance the taggability of the drugs to certain extent [9]. THQ represents one such class of modern phytochemicals which has been extensively studied and pathways of its molecular mechanisms have been comprehensively elucidated [6]. Utilization of these molecular targets coupled with suitable nanotechnological approaches can work wonders for the treatment of neurological, gastroenterological disorders and various oncological conditions.

\section{References}

1. Akhter S, Ahmad I, Ahmad MZ, Ramazani F, Singh A, et al. (2013) Nanomedicines as cancer therapeutics: current status. Curr. Cancer Drug Targets 13: 362-378.

2. Silva LM, Hill LE, Figueiredo E, Gomes CL (2014) Delivery of phytochemicals of tropical fruit by-products using poly (dl-lactide-co-glycolide) (PLGA) nanoparticles: Synthesis, characterization, and antimicrobial activity. Food Chem 165: 362-370.

3. Shakeri A, Sahebkar A (2015) Nanotechnology: A Successful Approach to Improve Oral Bioavailability of Phytochemicals. Recent Pat Drug Deliv Formul.

4. Wang S, Rui Su, Shufang Nie, Ming Sun, Jia Zhang, et al. (2014) Application of nanotechnology in improving bioavailability and bioactivity of diet-derived phytochemicals. J. Nutr. Biochem 25: 363-376.

5. Lee J, Park EY, Lee J (2013) Non-toxic nanoparticles from phytochemicals: preparation and biomedical application. Bioprocess Biosyst. Eng 37: 983-989.

6. Singh A, Iqbal Ahmad, Sohali A, Zaki Ahmad M, Zeenat lqbal (2012) Thymoquinone: Major Molecular Targets, Prominent Pharmacological Actions and Drug Delivery Concerns. Curr. Bioact. Compd 8: 334-344.

7. Singh A, Ahmad I, Akhter S, Jain GK, lqbal Z, et al. (2013) Nanocarrier based formulation of Thymoquinone improves oral delivery: Stability assessment, in vitro and in vivo studies. Colloids Surf. B Biointerfaces 102: 822-832.

8. Ahmad MZ, Sohali A, lqbal A, Singh A, Anwar M, et al. (2012) In vitro and in vivo evaluation of Assam Bora rice starch-based bioadhesive microsphere as a drug carrier for colon targeting. Expert Opin. Drug Deliv 9: 141-149.

9. Akhter S, Ahmad Z, Singh A, Ahmad I, Rahman M, et al. (2011) Cancer targeted metallic nanoparticle: targeting overview, recent advancement and toxicity concern. Curr Pharm Des 17: 1834-1850.
*Corresponding author: Anjali Singh, Department of Pharmaceutical Medicine Jamia Hamdard University, New Delhi-110062, India, Tel: +91-9891984500; Fax: +91 11 26059663; E-mail: anjali-singh.sch@jamiahamdard.ac.in

Received August 28, 2015; Accepted August 31, 2015; Published September 04, 2015

Citation: Singh A(2015) Phytochemical Nanomodification: Impending Perspectives. Next Generat Sequenc \& Applic 1: 118. doi:10.4172/2469-9853.1000118

Copyright: ( $) 2015$ Singh A. This is an open-access article distributed under the terms of the Creative Commons Attribution License, which permits unrestricted use, distribution, and reproduction in any medium, provided the original author and source are credited. 